\title{
Modern facilities in astronomy education
}

\section{Hayk Harutyunian and Areg Mickaelian}

Byurakan Astrophysical Observatory, Armenia email: hhayk_ast@yahoo.com

\begin{abstract}
Astronomical education is entering a new stage of development which is closely connected with the development of new technologies for communication, computing and data visualization. We discuss this evolution in the context of astronomy education in Armenia. As students spend only a short time in Byurakan Observatory for training in observations, they are not able to carry out systematic astronomical observations. Hence their training places emphasis on the use of astronomical archives and analysis of observational data obtained previously with the Byurakan telescopes and other ground-based and space telescopes. Thus, one of the aims of the Armenian Virtual Observatory is to support the training of students in this modern context.
\end{abstract}

Keywords. Astronomy education, Armenia

\section{Introduction}

Astronomical education in the world is entering a new stage of organization and development. This process is closely connected with the development of new technologies and technological diffusion in all the spheres of human activity, including education, enabling the use of entirely new possibilities in electronic communication, computing, visualization of observational data, new methods of data reduction, etc. Undoubtedly this process gives teachers many new favourable conditions for training their students. However, this trend also places completely new requirements on students in relation to their knowledge and skills, which must be be taken into account as well in the development of training programmes.

This progress is distinctly noticeable in Armenia and has resulted from several ongoing changes in the republic which, in their turn, dictate new approaches based mainly on technological developments. While suggesting many new possibilities, modern technologies at the same time require a higher level of knowledge. Although economic difficulties in the past decade (and the associated lack of opportunities for pursuing astronomy research) restricted the interest in astronomy among school pupils, the present young generation is gradually finding astronomy more and more attractive. Knowledge of computers and the Internet is the typical difference between present-day students and earlier generations of students. Thus, nowadays astronomy education requires heavy use of computer facilities and the Internet, just as it does in case of modern astronomical research. These facilities should be available for students as early as possible, since the sooner the students become proficient in the use of new technologies, the more efficient will be their future use of those technologies.

\section{Astronomy education in Armenia}

In Armenia there are sharp distinctions in the conditions in the universities and schools. Particularly noticeable differences exist in regard to access to modern technologies in the schools and universities. Only a small fraction of schools are accommodated with even the simplest modern facilities such as Internet and computers. School pupils use these 
facilities chiefly in Internet cafés, of which there are many in the large cities, but very few in the villages. So the students who enter university very often have entirely dissimilar starting knowledge on how to use computers and their accessories. The universities are better equipped with computers and are connected to the Internet by rather high-speed links.

During this period of rapid technological evolution astronomy educators have to confront the new educational reality presented by the realization of new technologies. This implies a certain risk of misunderstanding of the real meaning of astronomy on the part of students. Sometimes an exaggerated view of the role computers in astronomy (and in other sciences as well) becomes a danger for this science, transforming an astronomy student into the computer addict who simply dabbles with astronomical ideas or does a technician's work instead of using computers as a tool for serious scientific study. Thus, in using various technical devices one should always remember and teach the students that computers are not an end in themselves, but rather the means to facilitate mathematical or other operations for astronomical research.

In any given case a certain optimal solution should be found for training the students to use modern facilities as astronomers. Obviously the students need modern hardware such as powerful computers and Internet (including GRID technologies) as well as computing methods, usage of large astronomical databases, virtual observatories (VOs), etc. VOs, though not yet widely used, seem to be very efficient tools both for scientific research and for the training of students. The Armenian astronomy community, for example, has a unique database of the famous Markarian survey. The Digitized First Byurakan Survey (DFBS) is the digitized version of the spectroscopic survey completed for high galactic latitudes in 17,000 square degrees of the northern sky. The newly created Armenian Virtual Observatory (ArVO) contains the DFBS.

The new capabilities offered by such new facilities also indicate the need to train of a new breed of astronomy computing specialists who can drive modern research and make it much more efficient. Thus in 2005 we introduced a new graduate level course at the Yerevan State University called "Astronomical surveys, databases and virtual observatories." This course, which we believe is the first of its kind in the world, covers the modern understanding and treatment of large multi-wavelength data volumes.

As the students are not able to carry out systematic astronomical observations (they spend only a short time in Byurakan observatory for training in observations), our training emphasizes the usage of the astronomical archives and the reduction and analysis of previously obtained observational data, either from the Byurakan telescopes, or from other ground-based and space telescopes. The ArVO was thus created for such purposes as well. We are currently establishing a large data centre at the Armenian National Academy of Sciences, where a huge volume of astronomical data will be stored on the newly created ArmCluster supercomputer and students will have access to these data. A number of science projects will be carried out with these facilities, including both astronomical research and research on informatics methods. 